\title{
Fragment molecular orbital based interaction analyses on complexes between RBD variants and ACE2
}

\author{
Kazuki Akisawa ${ }^{1}$, Ryo Hatada ${ }^{1}$, Koji Okuwaki ${ }^{1}$, Shun Kitahara ${ }^{1}$, Yusuke Tachino ${ }^{1}$, \\ Yuji Mochizuki ${ }^{1,2 *}$ \\ 1) Department of Chemistry and Research Center for Smart Molecules, Faculty of \\ Science, Rikkyo University, 3-34-1 Nishi-ikebukuro, Toshima-ku, \\ Tokyo 171-8501, Japan \\ 2) Institute of Industrial Science, The University of Tokyo, 4-6-1 Komaba, Meguro-ku, \\ Tokyo 153-8505, Japan
}

Submitted to ChemRxiv on 2021/3/26, JST

Email: fullmoon@rikkyo.ac.jp (Y. Mochizuki:CA*) 


\begin{abstract}
The spike protein plays an important role in the infection of SARS-CoV-2 to human cells, and the binding affinity of receptor binding domain (RBD) to angiotensinconverting enzyme 2 (ACE2) is of special interest. In this report, we present a series of interaction analyses for the RBD - ACE2 complex (PDB ID: 6M0J) and mutated complexes of UK (B.1.1.7 lineage), South Africa (B1.351) and Brazil (B1.1.248) types, based on the fragment molecular orbital (FMO) calculations. The effects of mutations are investigated in terms of inter-fragment interaction energies (IFIEs), indicating the higher affinities of RBD variants with ACE2.
\end{abstract}


Severe acute respiratory syndrome coronavirus 2 (SARS-CoV-2) is the virus responsible for the 2019 coronavirus disease (COVID-19) first reported at Wuhan, China in December 2019. The issue of COVID-19 pandemic with devastating effects is still far from convergence or termination. The World Health Organization (WHO) Situation Report ${ }^{1}$ has reported the worldwide severe situations of cases and deaths continuously. Since SARS-CoV-2 is the first virus that human beings have faced, the lack of immunological experiences leads to a vulnerability to viral infection of COVID-19. In fact, SARS-CoV-2 is highly contagious with the number of regenerations ( $\mathrm{R}_{0}$ estimates $^{2,3}$ ) varying between about 2 and 5 .

SARS-CoV-2 has frequent mutations as influenza virus because of RNA virus. In particular, when mutations take place at the spike protein which commits the infection to human cell, such variants (or new lineages) may have higher infectious ability and escaping mechanism from neutralizing antibodies and vaccines. This concern has already been real, and several variants have been the main cause of epidemics. ${ }^{4}$ The mutation of Asp614Gly (or D614G) in the spike protein was found in April 2020, and this variant has spread over the world; several researches were reported on the enhanced replication and reproduction. ${ }^{5-8}$ In addition to the Asp614Gly mutation, variants with mutations at the receptor binding domain (RBD) (corresponding to Thr333-Pro527) ${ }^{9,10}$ have been 
identified since the latter half of 2020. RBD plays a central role in infection to human cells through the binding with the angiotensin-converting enzyme 2 (ACE2), and thus such variants have become the critical concern from a viewpoint of increased infectious power. Currently, the following three variants have attracted attentions. The UK variant (B.1.1.7 lineage) has Asn501Tyr (N501Y) mutation in RBD. Besides this mutation, the South Africa variant (B.1.351) and Brazil variant (B.1.1.248) take the mutation of Glu484Lys (E484K) in common, and the former and the latter have additional mutations of Lys417Asn (K417N) and Lys417Thr (K417T), respectively. ${ }^{11}$ Several reports ${ }^{12-15}$ pointed out enhanced contagious power and relatively high escaping ability from neutralizing antibodies or vaccines in these variants.

The fragment molecular orbital (FMO) method ${ }^{16-20}$ is of $a b$ initio (or non-empirical) type and has been applied to various biochemical systems. ${ }^{21-28}$ In Refs. 24-27, the influenza virus hemagglutinin (HA) was analyzed in terms of inter-fragment interaction energies (IFIEs) ${ }^{19-21}$ among amino acid residues in HA and antibody as well as sugar moieties, and potentially mutable residues could be identified. As FMO applications to SARS-CoV-2 related proteins, two complexes of 3CL main protease (Mpro) - N3 inhibiror, ${ }^{29-31}$ and RNA-dependent RNA polymerase - remdesivir ${ }^{32}$ as well as spike protein $^{33-36}$ have been investigated by using sets of IFIE and related quantities. Here, we 
report a series of interaction analyses for complexes formed between ACE2 and RBD with mutations ${ }^{11}$ (UK variant, South Africa variant and Brazil variant), based on the FMO calculations at up to the third-order Møller-Plesset perturbation (MP3) level. ${ }^{37}$ Mutual differences among RBD variants in interactions with ACE2 is a main interest of the present report.

The preparation of protein structures and the scheme of FMO calculations are summarized as follows. Residue-specific mutations were introduced to the 6M0J (PDB ID $)^{10}$ structure formed between ACE2 and Wuhan type RBD. With the MOE software, ${ }^{38}$ the positions of hydrogen atoms were optimized with molecular mechanics (MM) with the AMBER10:EHT force field. ${ }^{39}$ The total number of residues in 6M0J was 784, and all 80 water molecules in the original record were retained; no water molecule was observed in the RBD - ACE2 interfacial region. The respective pointwise mutations for amino acid residues were made with MOE for the three variants of UK (B.1.1.7 lineage), South Africa (B.1.351), and Brazil (B.1.1.248). ${ }^{11}$ For these naively mutated structures, an MM based optimization (AMBER10:EHT) was done on the mutated points without a tether mask. Then, full optimizations with a tether $=1.0$ condition were performed for the RBD side; this protocol was applied to the mutation-less Wuhan structure as well; the final structure set was slightly different from that used in Ref. 33. Fig. 1 illustrates the interfacial regions 
of processed structures, where the mutated residues in RBD such as Asn501Tyr and Lys417Asn are labeled with red font. From this figure, changes due to the mutations are expectable because of alternations of both electrostatic and dispersion type interactions between RBD and ACE2. The mutation of Lys417 committing the salt-bridge formation with Asp30 in ACE2 should be of special interest. ${ }^{40}$

The ABINIT-MP program ${ }^{18,20}$ was used for two-body FMO calculations of MP2 (second-order MP perturbation) ${ }^{41-43}$ and MP3. ${ }^{37}$ A partially renormalized MP2 (PRMP2) ${ }^{44}$ was used to make overestimations in interaction energies by MP2. The energy corrections by these correlated treatments are usually used as the "DI" term indicating "dispersion" in the energy decomposition analysis of IFIE termed as PIEDA ${ }^{45,46}$; "ES" (electrostatic), "EX" (Pauli exclusion repulsion)", and "CT" (charge transfer plus mix effects) are evaluated at the Hartree-Fock (HF) level. It should be noted that the electron correlation can introduce not only the attractive interaction of dispersion type but also the energy lowering through reductions of excess ionic characters in the HF description. For a further separation of "DI" term, the local response dispersion (LRD) ${ }^{47}$ calculation was employed as a so-called "HF+D" approach of FMO. ${ }^{48}$ LRD could estimate stabilization energies of pure dispersion type through the higher-order multipole expansions of density. A "DI" term would be considered as a sum of "LRD" and "Erest." (related with reductions 
of excess iconicity or electron localization). As shown in Refs. 33 and 49, the MP2.5

scaling (in which the MP3 incremental correlation energy is halved and added to the MP2 energy $)^{50}$ could work better than does the straight MP3 in evaluating interaction energies comparable to those obtained by the costly but reliable coupled cluster singles and doubles with perturbative triples $(\operatorname{CCSD}(\mathrm{T})) .{ }^{51} \mathrm{MP} 2.5$ was thus adopted as our best-effort recipe. The $6-31 \mathrm{G}^{* 52}$ and cc-pVDZ ${ }^{53}$ basis sets were used, where the latter could be more flexible in the description of electronic structures. The number of fragments including water molecules was 864 throughout the four RBD - ACE2 models (Wuhan, UK, South Africa and Brazil). All the FMO calculations were performed by the massively parallel computing resource of supercomputer Fugaku as in the case of previous study. ${ }^{33}$ A typical timing was $4.5 \mathrm{~h}$ for a single FMO-MP3/cc-pVDZ job with 384 nodes (or 1 rack) of Fugaku.

Below we give the results and discussion. The numerical results obtained at the respective correlation levels with 6-31G* and cc-pVDZ basis sets are given in Fig. S1 and Table S1 of Electronic Supporting Information (ESI). For a simplicity of main text, the following presentation and discussion are based on the values evaluated by the MP2.5/cc-pVDZ method. Fig. 2 indicates the total IFIE values of RBD - ACE2 interactions. As can be seen from this figure, the UK variant has a slightly larger value of 
$-928.6 \mathrm{kcal} / \mathrm{mol}$ than $-909.5 \mathrm{kcal} / \mathrm{mol}$ of the original Wuhan case (1.03 times larger), and the South Africa and Braizl variants provide sizable larger values of $-1108.4 \mathrm{kcal} / \mathrm{mol}$ and $-1106.8 \mathrm{kcal} / \mathrm{mol}$, respectively (1.23 times). In Ref. 34, a similar increment of about 10 $\mathrm{kcal} / \mathrm{mol}$ for RBD - ACE2 interaction energy was estimated for the UK variant relative to Wuhan.

The results of differences in IFIE ( $\triangle$ IFIE) for interfacial residues (summed up counter residues in RBD or ACE2) are plotted for the three variants in Fig. S2 of ESI, where the values of original Wuhan are used as a reference set. Positive and negative values of $\Delta$ IFIE correspond to destabilizations and stabilizations, respectively, due to the mutations. For the UK variant, Tyr501 (after the mutation) in RBD shows $-35.3 \mathrm{kcal} / \mathrm{mol}$, and Tyr41 and Lys353 in ACE2 have small negative values. Similar stabilizations for Tyr501 are found for both South Africa and Brazil variants. There are considerable destabilizations (about $300 \mathrm{kcal} / \mathrm{mol}$ ) by Lys417Asn (South Africa) and Lys417Thr (Brazil). This loss of electrostatic stabilizations is overcompensated with $-446.4 \mathrm{kcal} / \mathrm{mol}$ (South Africa) by the Glu484Lys mutation; the charge situation is reversed. Asp30 in ACE2 is destabilized because of the lack of salt-bridge with Lys417. ${ }^{33,40}$ It is also notable that Glu35 and Glu75 in ACE2 are rather stabilized. In other words, the role of Lys417 in Wuhan RBD is replaced by Lys484 in the South Africa and Brazil variants. 
Next, the results of pairs of residue in RBD and residue in ACE2 are focused on. Fig. 3 shows the IFIEs (whose absolute values are larger than $10 \mathrm{kcal} / \mathrm{mol}$ ) of these interresidue pairs for the three mutants. In this figure, the label " $\mathrm{A}$ " corresponds to the zone related with the residue 417 (Lys, Asn, or Thr). Similarly, the labels "B" and "C" correlate with the zones around residue 484 (Glu or Lys) and residue 501 (Asn or Tyr), respectively. About zone A, the UK variant has almost the same values as in the original Wuhan, indicating the importance of the salt-bridge $(-126.9 \mathrm{kcal} / \mathrm{mol})$ between Lys417 and Asp30. Note that Lys417 is also crucial in interacting with the B38 Fab antibody. ${ }^{33,54}$ It is noteworthy that there are electrostatic repulsions with Lys31 and His34 in ACE2. In the South Africa and Brazil variants, these repulsions are gone because of the replacement of Lys417, contributing to the gross increment of IFIE (indicated in Fig. 2) relative to the UK variant (and also non-mutated Wuhan).

The Glu484Lys mutation (South Africa and Brazil) in zone B (see Fig.3 again) changes the situation from attraction $(-76.8 \mathrm{kcal} / \mathrm{mol})$ to repulsion $(36.3 \mathrm{kcal} / \mathrm{mol}$ (South Africa)) with Lys31 in ACE2 but provides a sizable stabilization with Glu75 in ACE2. Namely, the role of Glu484 - Lys31 pair is replaced by the Lys484 - Glu75 pair in the South Africa and Brazil mutants. The stabilization is mainly attributed to the electrostatic ("ES") in nature, as plotted in Fig. S3 of ESI of the PIEDA results; see the Glu484 - Lys31 pair in 
the windows of Wuhan and UK and also Lys484 - Glu75 pair in the windows of South Africa and Brazil. Because ACE2 is sizably charged in negative due to the rich presence of Glu (as many as 47) and Asp (similarly 33), the Glu484Lys mutation in RBD should increment overall attractive interactions with ACE2; refer to Fig. 2 again.

The Asn501Tyr mutation of zone C takes place throughout the three variants (Fig. 3), and notable increments in stabilization are observed for the pairs associated with Tyr41 and Lys353. This observation is in accord with results of the UK variant in Ref. 34. The increased "DI" contribution (by PIEDA) is responsible to increments by the Tyr replacement as illustrated in Fig. S3.

To understand the "DI" stabilization in more detail, it is divided to the components of "LRD" and "Erest." as plotted in Fig. S4 of ESI. It can be seen that the balance of these two components differs for the respective pairs. "Erest." dominates for the charged pair of Lys417 - Asp30 (salt-bridge in Wuhan and UK), to reduce the excess ionicity at the HF level. In contrast, the pair of charged residue and neutral residue, e.g., Tyr501 - Lys352 (in South Africa and Brazil), has almost half each. For the neutral pair such as Tyr501 Tyr41, "LRD" is leading as expected. As a whole, (pure) dispersion type interactions play a key role in incrementing stabilizations associated with the Asn501Tyr mutation of the UK, South Africa and Brazil variants. 
A recent report ${ }^{55}$ made an alert that the contagion abilities of the UK and South Africa variants are 9 times and 3 times large relative to the original Wuhan without mutation. These relations are not directly related to the relative strengths of IFIE stabilizations, because we used only the RBD - ACE2 complex model. Nonetheless, some more consideration might be useful as follows. FMO calculations have a tendency to overestimate IFIE values dominated by "ES" contributions even for distant pairs of both charged fragments, and thus a statistical screening ${ }^{56}$ or Yukawa decay factor ${ }^{57}$ have sometimes been employed. On the other hand, it has been known that an effective range of electrostatic interactions in proteins is $10-15 \AA . .^{58-60}$ We thus attempted partial summations of IFIE from $3 \AA$ to $10 \AA$ (step $1 \AA$ ) at the interfacial region, by increasing the residue pairs between RBD and ACE2; no decay modification was used. The resulted plots are given in Fig. S5 of ESI. At $5 \AA$ A, the summed values of Wuhan, UK, South Africa and Brazil are $-412.0 \mathrm{kcal} / \mathrm{mol},-424.0 \mathrm{kcal} / \mathrm{mol},-312.3 \mathrm{kcal} / \mathrm{mol}$ and $-339.0 \mathrm{kcal} / \mathrm{mol}$, respectively. Notably, the UK variant (almost) always has larger IFIE sums relative to the original Wuhan, suggesting a short-range interaction assistance by Asn501Tyr. The South Africa and Brazil variants shows larger values after $7 \AA$ than do the Wuhan and UK cases. At $10 \AA$, the summed values are $-398.3 \mathrm{kcal} / \mathrm{mol},-417.5 \mathrm{kcal} / \mathrm{mol},-469.0 \mathrm{kcal} / \mathrm{mol}$ and $439.9 \mathrm{kcal} / \mathrm{mol}$, in the above-listed order. Such distance-dependent changes in 
stabilization situations may be attributed to the two mutations occurred at Lys417 and Glu484 in the South Africa and Brazil mutants.

Furthermore, we tried a computational experiment that the mutations of Glu484Lys and Asn501Tyr take place under keeping Lys417 unchanged. The corresponding model set-up and FMO calculation were made, and the total IFIE between this mutated RBD and ACE2 was estimated to be $-1377.1 \mathrm{kcal} / \mathrm{mol}$. This value is 1.53 times larger than the original Wuhan without mutation, implying a further enhanced affinity with ACE2 relative to the three variants investigated so far. The possibilities of various mutations were investigated with machine learning based cluster analyses in Ref. 61, and such an approach would provide potential targets to be calculated by FMO.

In summary, a series of FMO calculations were performed for $6 \mathrm{M} 0 \mathrm{~J}^{10}$ derived complexes between ACE2 and RBD with and without mutations. The MP2.5/cc-pVDZ treatment estimated that the incremental factor of sum of IFIEs between RBD and ACE2 is 1.03 for the UK variant (B.1.1.7 lineage) relative to the Wuhan case and that its factor is increased to 1.23 for the South African (B1.351) and Brazilian (B1.1.248) variants. Detailed analyses in interactions due to residue-specific mutations such as Asn501Tyr (N501Y) and Glu484Lys (E484K) were made as well. Our ab initio theoretical results were in line with experimentally known facts ${ }^{12-15,55}$ for the UK, South Africa and Brazil 
variants. We hope the FMO based analyses will provide useful insights for the nature of interactions associated with the spike protein (with and without mutations). ${ }^{33-36}$

YM would thank Dr. Yuto Komeiji and Prof. Shigenori Tanaka for their discussion and encouragement. As in the previous studies, ${ }^{30,33}$ computational resources of the Fugaku supercomputer (the evaluation environment in the trial phase) were provided by the RIKEN Center for Computational Science (R-CCS) under a special project against COVID-19 from April 2020. The present work was supported by Rikkyo SFR.

\section{Conflicts of interest}

There are no conflicts to declare. 


\section{References}

1 WHO, https://www.who.int/emergencies/diseases/novel-coronavirus2019/situation-reports (accessed on 2021/3/26).

2 K. Linka, M. Peirlinck and E. Kuhl, Comput. Mech., 2020, 66, 1035-1050.

3 A. C. Walls, Y. J. Park, M. A. Tortorici, A. Wall, A. T. McGuire and D. Veesler, Cell, 2020, 181, 281-292.e6.

4 B. Korber, W. M. Fischer, S. Gnanakaran, H. Yoon, J. Theiler, W. Abfalterer, N. Hengartner, E. E. Giorgi, T. Bhattacharya, B. Foley, K. M. Hastie, M. D. Parker, D. G. Partridge, C. M. Evans, T. M. Freeman, T. I. de Silva, A. Angyal, R. L. Brown, L. Carrilero, L. R. Green, D. C. Groves, K. J. Johnson, A. J. Keeley, B. B. Lindsey, P. J. Parsons, M. Raza, S. Rowland-Jones, N. Smith, R. M. Tucker, D. Wang, M. D. Wyles, C. McDanal, L. G. Perez, H. Tang, A. Moon-Walker, S. P. Whelan, C. C. LaBranche, E. O. Saphire and D. C. Montefiori, Cell, 2020, 182, 812-827.e19.

5 S. Isabel, L. Graña-Miraglia, J. M. Gutierrez, C. Bundalovic-Torma, H. E. Groves, M. R. Isabel, A. R. Eshaghi, S. N. Patel, J. B. Gubbay, T. Poutanen, D. S. Guttman and S. M. Poutanen, Sci. Rep., 2020, 10, 1-9.

6 S. M. C. Gobeil, K. Janowska, S. McDowell, K. Mansouri, R. Parks, K. Manne, 
V. Stalls, M. F. Kopp, R. Henderson, R. J. Edwards, B. F. Haynes and P. Acharya, Cell Rep., 2021, 34, 108630.

A. Fernández, ACS Med. Chem. Lett., 2020, 11, 1667-1670.

B. Zhou, T. Thi, N. Thao, D. Hoffmann, A. Taddeo, N. Ebert, F. Labroussaa, A.

Pohlmann, J. King, S. Steiner, J. N. Kelly, J. Portmann, N. J. Halwe, U. Lorenz,

S. Trüeb, X. Fan, B. Hoffmann, L. Wang, L. Thomann, X. Lin, H. Stalder, B.

Pozzi, T. J. Stark, J. R. Barnes, R. Dijkman, J. Jores, C. Benarafa and S.- Dg, Nature, 2021, DOI:10.1038/s41586-021-03361-1.

9 F. Li, W. Li, M. Farzan and S. C. Harrison, Science., 2005, 309, 1864-1868.

10 J. Lan, J. Ge, J. Yu, S. Shan, H. Zhou, S. Fan, Q. Zhang, X. Shi, Q. Wang, L. Zhang and X. Wang, Nature, 2020, 581, 215-220.

11 Science Brief: Emerging SARS-CoV-2 Variants, https://www.cdc.gov/coronavirus/2019-ncov/more/science-andresearch/scientific-brief-emerging-variants.html. (2021/3/6)

12 D. Planas, T. Bruel, L. Grzelak, F. Guivel-Benhassine, I. Staropoli, F. Porrot, C. Planchais, J. Buchrieser, M. M. Rajah, E. Bishop, M. Albert, F. Donati, S. Behillil, V. Enouf, M. Maquart, M. Gonzalez, J. De Sèze, H. Péré, D. Veyer, A. Sève, E. Simon-Lorière, S. Fafi-Kremer, K. Stefic, H. Mouquet, L. Hocqueloux, 
S. van der Werf, T. Prazuck and O. Schwartz, bioRxiv, 2021, preprint, DOI:

2021.02.12.430472.

13 S. A. Madhi, V. Baillie, C. L. Cutland, M. Voysey, A. L. Koen, L. Fairlie, S. D.

Padayachee, K. Dheda, S. L. Barnabas, Q. Ebrahim Bhorat, C. Briner, G. Kwatra,

W.-V. COVID team, K. Ahmed, P. Aley, S. Bhikha, J. N. Bhiman, A. Ebrahim

Bhorat, J. du Plessis, A. Esmail, M. Groenewald, E. Horne, S.-H. Hwa, A. Jose,

T. Lambe, M. Laubscher, M. Malahleha, M. Masenya, M. Masilela, S.

McKenzie, K. Molapo, A. Moultrie, S. Oelofse, F. Patel, S. Pillay, S. Rhead, H.

Rodel, L. Rossouw, C. Taoushanis, H. Tegally, A. Thombrayil, S. van Eck, C.

Kurt Wibmer, N. M. Durham, E. J. Kelly, S. Madhi, A. Izu, C. B. Hill Hons, V.

Baillie, G. Kwatra, A. Koen MBBCh, S. Bhikha MBBCh, C. Taoushanis

BPharm, A. Moultrie Hons, A. Thombrayil MBBCh, S. McKenzie, J. du Plessis

Hons, M. Laubscher and S. S. Africa Madhi, Alex Sigal, 2021, 13, 2.

14 V. Monique, C. Xinyue, S. A. Gilles, M. O. Mohsen, D. E. Speiser and M. F.

Bachmann, bioRxiv, 2021, prepeint, DOI:10.1101/2021.03.04.433887.

15 M. Hendy, S. Kaufman and M. Ponga, bioRxiv, 2021, preprint,

DOI:10.1101/2021.03.04.433970.

16 K. Kitaura, E. Ikeo, T. Asada, T. Nakano and M. Uebayasi, Chem. Phys. Lett., 
$1999, \mathbf{3 1 3}, 701-706$.

17 D. Fedorov and K. Kazuo, The Fragment Molecular Orbital Method: Practical Applications to Large Molecular Systems, CRC Press, 2009.

18 Y. Mochizuki, T. Nakano, K. Sakakura, Y. Okiyama, H. Watanabe, K. Kato, Y.

Akinaga, S. Sato, J. Yamamoto, K. Yamashita, T. Murase, T. Ishikawa, Y.

Komeiji, Y. Kato, N. Watanabe, T. Tsukamoto, H. Mori, K. Okuwaki, S. Tanaka,

A. Kato, C. Watanabe and K. Fukuzawa, in Y. Mochizuki, S. Tanaka and K.

Fukuzawa ed.,Recent Advances of the Fragment Molecular Orbital Method,

Springer Singapore, 2021, 53-67.

19 D. G. Fedorov, T. Nagata and K. Kitaura, Phys. Chem. Chem. Phys., 2012, 14, $7562-7577$

20 S. Tanaka, Y. Mochizuki, Y. Komeiji, Y. Okiyama and K. Fukuzawa, Phys.

Chem. Chem. Phys., 2014, 16, 10310-10344.

21 S. Amari, M. Aizawa, J. Zhang, K. Fukuzawa, Y. Mochizuki, Y. Iwasawa, K.

Nakata, H. Chuman and T. Nakano, J. Chem. Inf. Model., 2006, 46, 221-230.

22 K. Fukuzawa, Y. Mochizuki, S. Tanaka, K. Kitaura and T. Nakano, J. Phys.

Chem. B, 2006, 110, 16102-16110.

23 Y. Sheng, H. Watanabe, K. Maruyama, C. Watanabe, Y. Okiyama, T. Honma, K. 
Fukuzawa and S. Tanaka, Comput. Struct. Biotechnol. J., 2018, 16, 421-434.

24 T. Iwata, K. Fukuzawa, K. Nakajima, S. Aida-Hyugaji, Y. Mochizuki, H.

Watanabe and S. Tanaka, Comput. Biol. Chem., 2008, 32, 198-211.

25 S. Anzaki, C. Watanabe, K. Fukuzawa, Y. Mochizuki and S. Tanaka, J. Mol.

Graph. Model., 2014, 53, 48-58.

26 K. Takematsu, K. Fukuzawa, K. Omagari, S. Nakajima, K. Nakajima, Y.

Mochizuki, T. Nakano, H. Watanabe and S. Tanaka, J. Phys. Chem. B, 2009,

113, 4991-4994.

27 A. Yoshioka, K. Fukuzawa, Y. Mochizuki, K. Yamashita, T. Nakano, Y.

Okiyama, E. Nobusawa, K. Nakajima and S. Tanaka, J. Mol. Graph. Model., 2011, 30, 110-119.

28 F. Xu, S. Tanaka, H. Watanabe, Y. Shimane, M. Iwasawa, K. Ohishi and T.

Maruyama, Viruses., 2018, 10, 236.

29 R. Hatada, K. Okuwaki, Y. Mochizuki, Y. Handa, K. Fukuzawa, Y. Komeiji, Y.

Okiyama and S. Tanaka, J. Chem. Inf. Model., 60, 3593-3602.

30 R. Hatada, K. Okuwaki, K. Akisawa, Y. Mochizuki, Y. Handa, K. Fukuzawa, Y.

Komeiji, Y. Okiyama and S. Tanaka, Appl. Phys. Express, 14, 27003.

31 S. Tanaka, S. Tokutomi, R. Hatada, K. Okuwaki, K. Akisawa, K. Fukuzawa, Y. 
Komeiji, Y. Okiyama and Y. Mochizuki, ChemRxiv, 2021, preprint, DOI:

10.26434/chemrxiv.13775182.v1.

32 K. Kato, T. Honma and K. Fukuzawa, J. Mol. Model. Graph., 2020, 100, 107695.

33 K. Akisawa, R. Hatada, K. Okuwaki, Y. Mochizuki, K. Fukuzawa, Y. Komeiji and S. Tanaka, RSC Adv., 2021, 11, 3272-3279.

34 C. Watanabe, Y. Okiyama, S. Tanaka, K. Fukuzawa and H. Teruki, Chem. Sci., 2021, DOI: 10.1039/D0SC06528E

35 H. Lim, A. Baek, J. Kim, M. S. Kim, J. Liu, K. Y. Nam, J. H. Yoon and K. T. No, Sci. Rep., 2020, 10, 1-8.

36 K. Watanabe, C. Watanabe, T. Honma, Y. Tian, Y. Kawashima, N. Kawashita, T.Takagi and K. Fukuzawa, ChemRxiv, 2021, preprint, DOI: 10.26434/chemrxiv.14135138.v1.

37 Y. Mochizuki, K. Yamashita, K. Fukuzawa, K. Takematsu, H. Watanabe, N. Taguchi, Y. Okiyama, M. Tsuboi, T. Nakano and S. Tanaka, Chem. Phys. Lett., 2010, 493, 346-352.

38 Molecular Operating Environment (MOE),Montreal, QC, Canada, 2013. Group Inc., https://www.chemcomp.com/Products.htm

39 D. A. Case, T. E. Cheatham, T. Darden, H. Gohlke, R. Luo, K. M. Merz, A. 
Onufriev, C. Simmerling, B. Wang and R. J. Woods, J. Comput. Chem., 2005, 26, 1668-1688.

40 B. Luan and T. Huynh, bioRxiv, 2021, preprint, DOI:

10.1101/2021.02.06.430088.

41 Y. Mochizuki, T. Nakano, S. Koikegami, S. Tanimori, Y. Abe, U. Nagashima and K. Kitaura, 112, 442-452.

42 Y. Mochizuki, K. Yamashita, T. Murase, T. Nakano, K. Fukuzawa, K.

Takematsu, H. Watanabe and S. Tanaka, Chem. Phys. Lett., 2008, 457, 396-403.

43 Y. Mochizuki, S. Koikegami, T. Nakano, S. Amari and K. Kitaura, Chem. Phys. Lett., 2004, 396, 473-479.

44 C. E. Dykstra and E. R. Davidson, Int., J. Quantum Chem., 2000, 78, 226-236.

45 D. G. Fedorov and K. Kitaura, J. Comput. Chem.,2006 28, 222-237.

46 T. Tsukamoto, K. Kato, A. Kato, T. Nakano, Y. Mochizuki and K. Fukuzawa, J. Comput. Chem. Jpn, 2015, 14, 1-9.

47 T. Sato and H. Nakai, J. Chem. Phys., 2009, 131, 224104.

48 Y. Akinaga, K. Sakakura, K. Akisawa, R. Hatada, T. Abe, K. Okuwaki and Y. Mochizuki, to be Submitted.

49 H. Yamada, Y. Mochizuki, K. Fukuzawa, Y. Okiyama and Y. Komeiji, Comput. 
Theor. Chem., 2017, 1101, 46-54.

50 M. Pitoňák, P. Neogrády, J. Černý, S. Grimme and P. Hobza, ChemPhysChem, 2009, 10, 282-289.

51 K. Raghavachari, G. W. Trucks, J. A. Pople, M. Head-Gordon, Chem. Phys. Lett., 1989, 157, 479-483.

52 J. B. Foresman and A. Frisch, Exploring chemistry with electronic structure methods, 2nd edition, Gaussian Inc., Pittsburgh, 1996.

53 D. E. Woon and T. H. Dunning, J. Chem. Phys., 1995, 103, 4572-4585.

54 Y. Wu, F. Wang, C. Shen, W. Peng, D. Li, C. Zhao, Z. Li, S. Li, Y. Bi, Y. Yang, Y. Gong, H. Xiao, Z. Fan, S. Tan, G. Wu, W. Tan, X. Lu, C. Fan, Q. Wang, Y. Liu, C. Zhang, J. Qi, G. F. Gao, F. Gao and L. Liu, Science 2020, 368, $1274-$ 1278.

55 C. Laffeber, K. de Koning, R. Kanaar and J. H. Lebbink, bioRxiv, 2021, preprint, DOI: $10.1101 / 2021.02 .22 .432357$

56 S. Tanaka, C. Watanabe and Y. Okiyama, Chem. Phys. Lett., 2013, 556, 272277.

57 B. Drobot, M. Schmidt, Y. Mochizuki, T. Abe, K. Okuwaki, F. Brulfert, S. Falke, S. A. Samsonov, Y. Komeiji, C. Betzel, T. Stumpf, J. Raff and S. Tsushima, 
Phys. Chem. Chem. Phys., 2019, 21, 21213-21222.

58 H. X. Zhou and X. Pang, Chem. Rev., 2018, 118, 1691-1741.

59 K. K. Lee, C. A. Fitch and B. García-Moreno E., Protein Sci., 2002, 11, 1004 1016.

60 R. O. Louro, T. Catarino, C. M. Paquete and D. L. Turner, FEBS Lett., 2004, 576, $77-80$.

61 J. Chen, R. Wang, M. Wang and G.-W. Wei, J. Mol. Biol., 2020, 432, 5212-5226. 

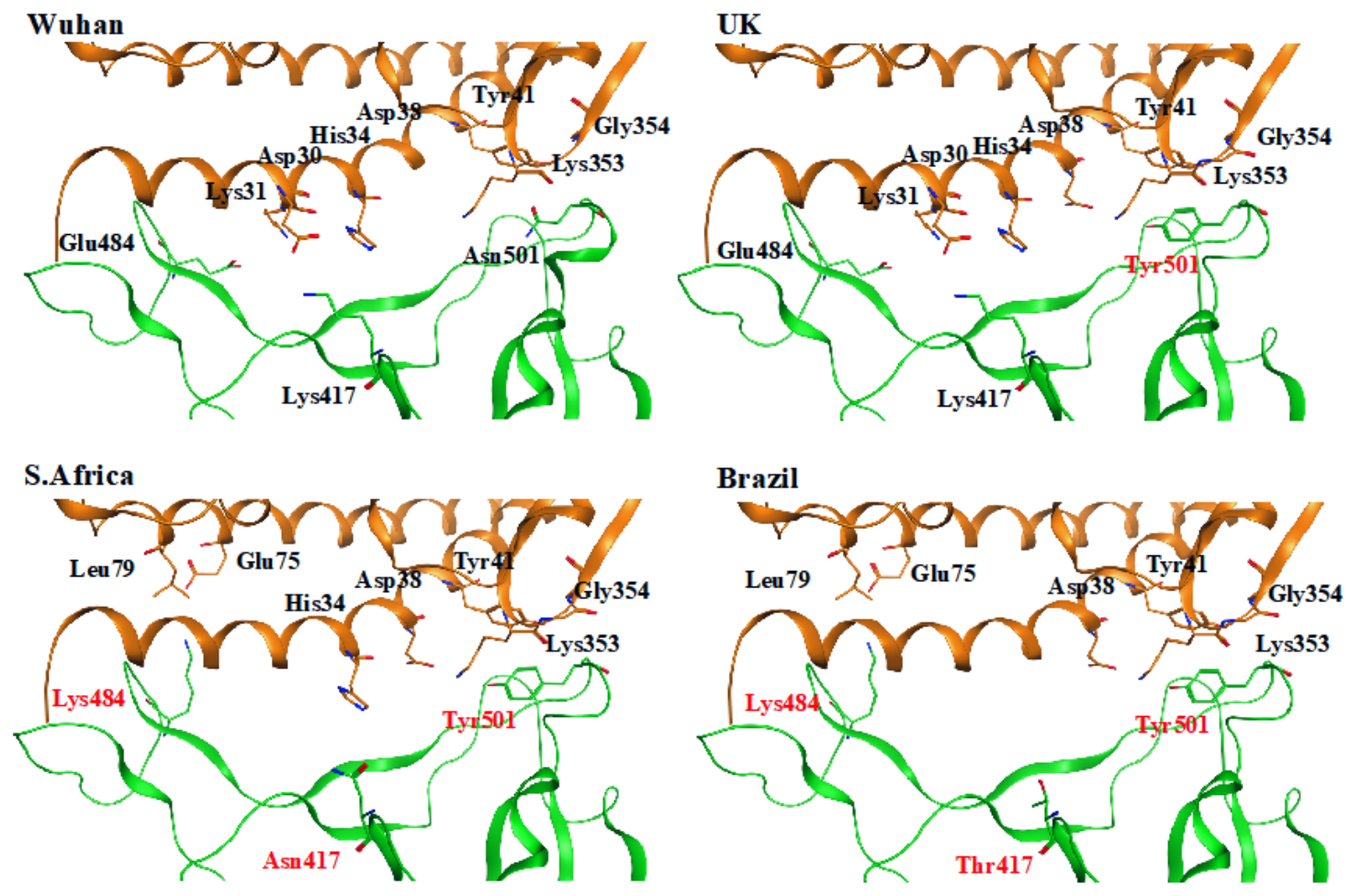

Fig. 1. Illustrations of RBD - ACE2 interfacial region for four models (Wuhan, UK, South

Africa and Brazil). Green and orange colors are assigned to identify RBD and ACE2,

respectively. Residues indicated with red font correspond to mutations. Ball-and-stick representation is used for residues of interest. 


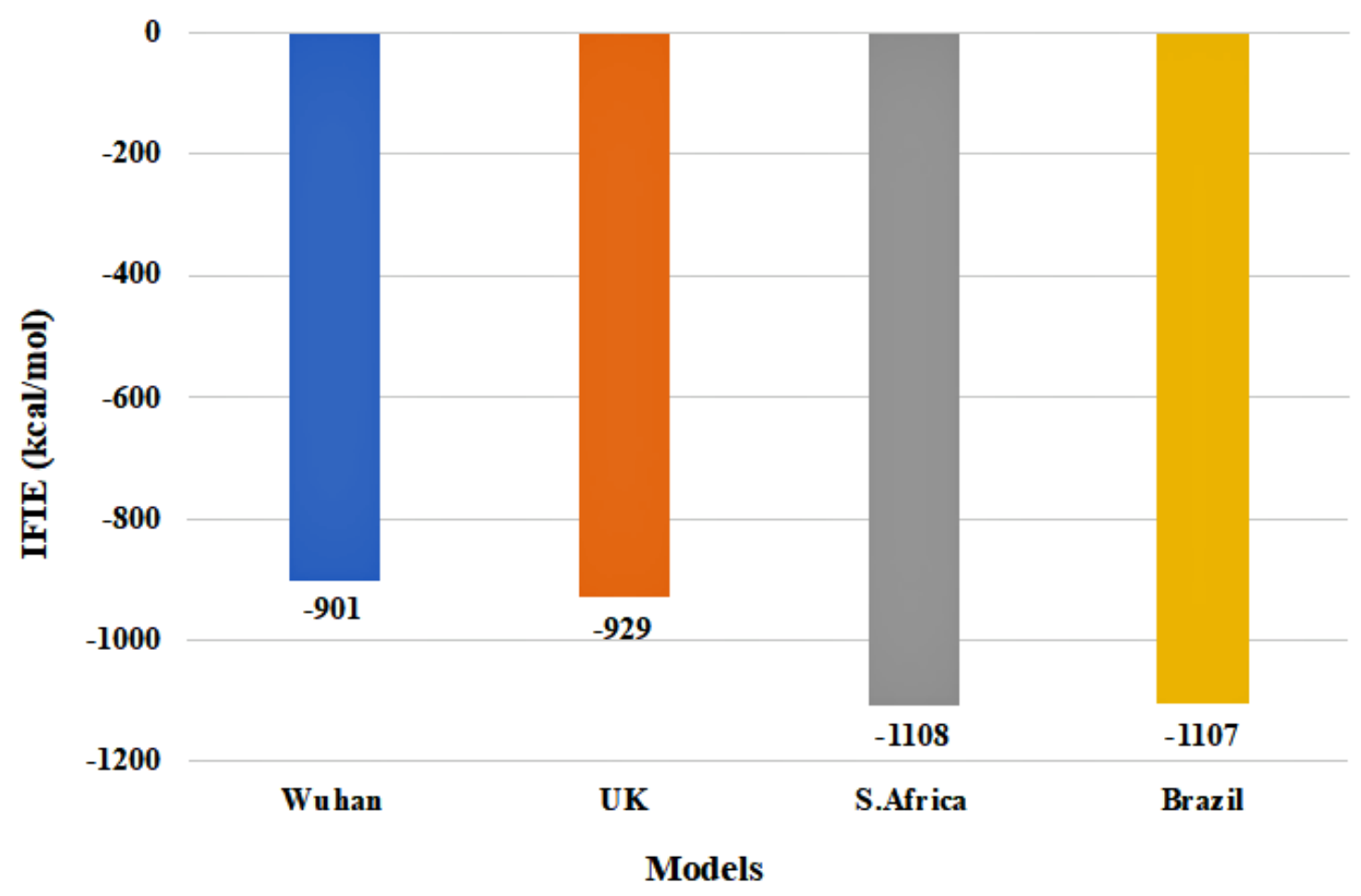

Fig. 2. Plots of total IFIE values RBD - ACE2 interactions evaluated by MP2.5/cc-pVDZ method (as a best-effort treatment) for four models (Wuhan, UK, South Africa and Brazil).

Numerical values are found in Table S1 of ESI. 

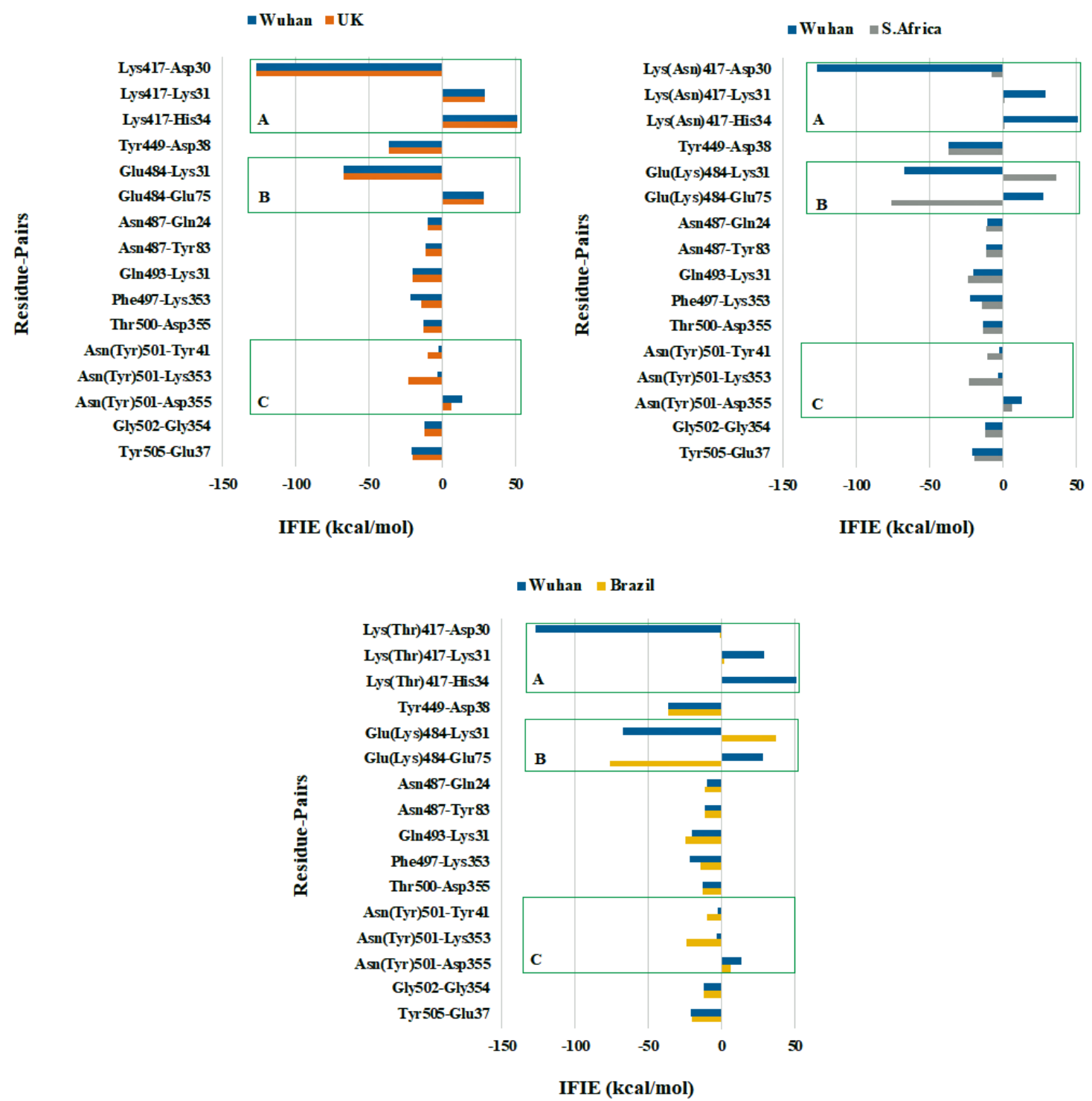

Fig. 3. Comparative plots of IFIE values (whose absolute values are larger than 10 $\mathrm{kcal} / \mathrm{mol}$ ) between RBD residues and ACE2 residues for three variants (UK, South Africa and Brazil). Results of Wuhan are plotted as reference guide. Numerical values are found in IFIE column in Tables S4 - S7 of ESI. See main texts for zones of A, B and C. 


\section{Electronic Supporting Information}

\section{Fragment molecular orbital based interaction analyses on complexes between RBD variants and ACE2}

Kazuki Akisawa ${ }^{1}$, Ryo Hatada ${ }^{1}$, Koji Okuwaki ${ }^{1}$, Shun Kitahara ${ }^{1}$, Yusuke Tachino ${ }^{1}$, Yuji Mochizuki ${ }^{1,2 *}$

1) Department of Chemistry and Research Center for Smart Molecules, Faculty of Science, Rikkyo University, 3-34-1 Nishi-ikebukuro, Toshima-ku,

Tokyo 171-8501, Japan

2) Institute of Industrial Science, The University of Tokyo, 4-6-1 Komaba, Meguro-ku, Tokyo 153-8505, Japan

Submitted to ChemRxiv on 2021/3/26, JST

Email: fullmoon@rikkyo.ac.jp (Y. Mochizuki: CA*) 


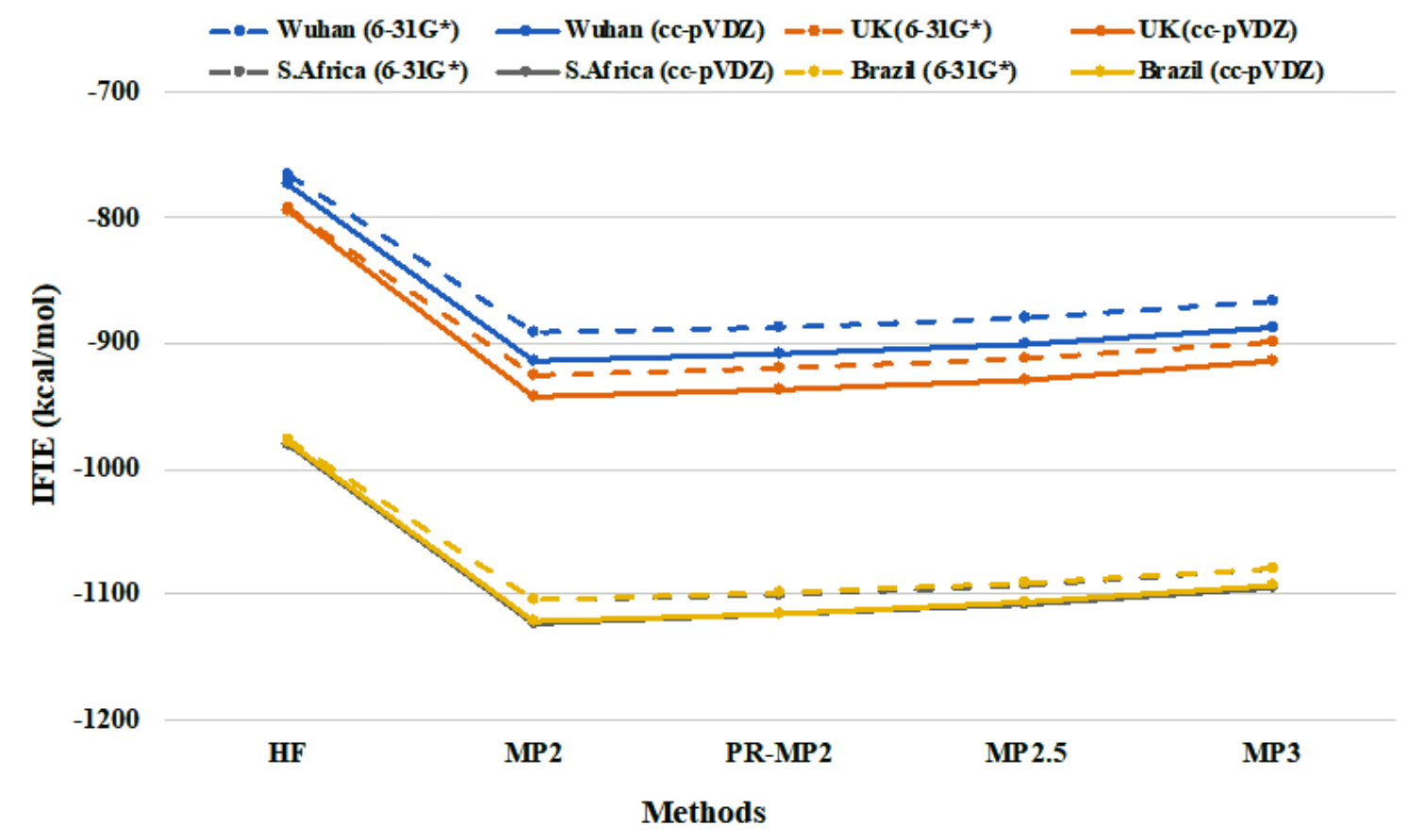

Fig. S1. Plots of total IFIE values of RBD - ACE2 interactions evaluated by various methods (see main texts). Numerical values are compiled in Table S1. Short notation of "S.Africa" is frequently used for South Africa variant in Fig. and Table hereafter. 
Diff. (UK - Wuhan) 1@RBD - all@ACE2

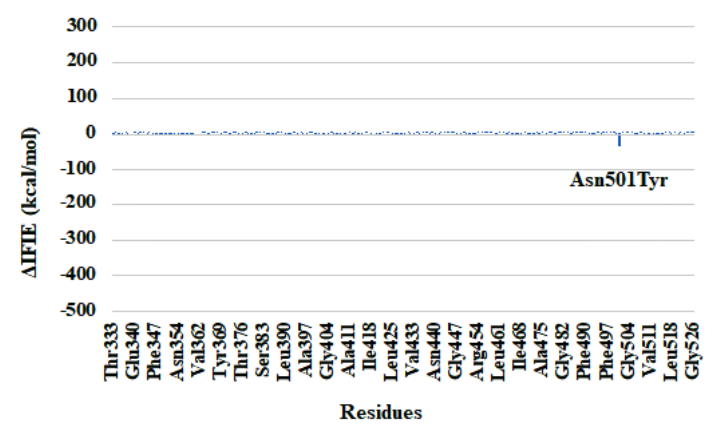

Diff. (S.Africa - Wuhan) 1@RBD - all@ACE2

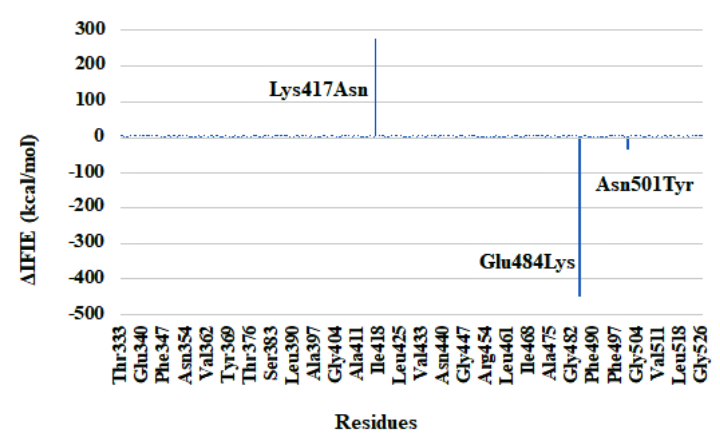

Diff. (Brazil - Wuhan) 1@RBD - all@ACE2

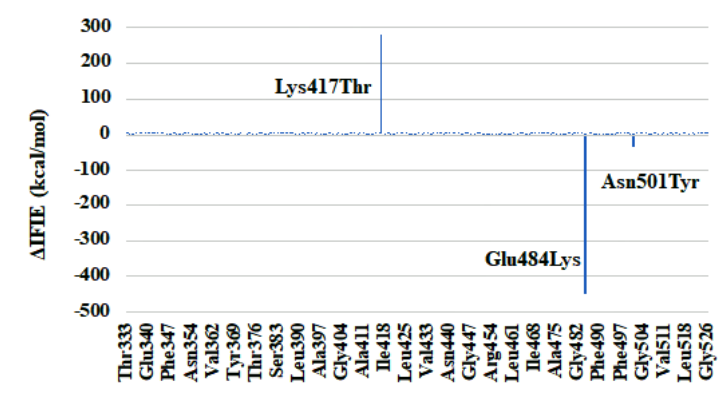

Residues
Diff. (UK - Wuhan) 1@ACE2 - all@RBD

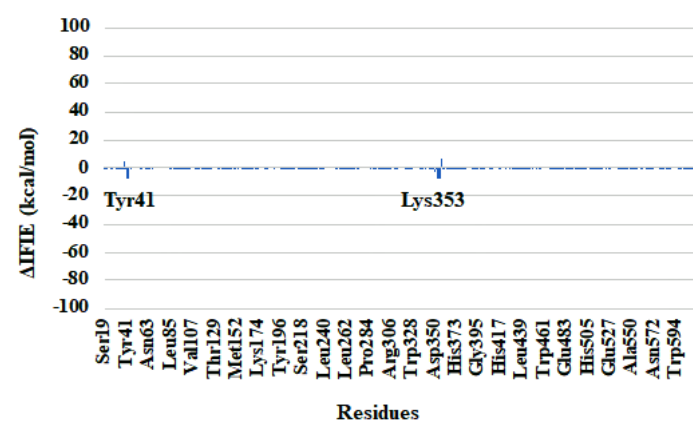

Diff. (S.Africa - Wuhan) 1@ACE2 - all@RBD

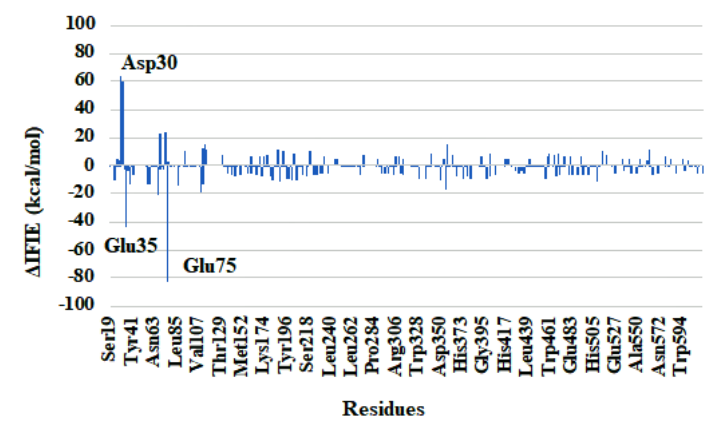

Diff. (Brazil - Wuhan) 1@ACE2 - all@RBD

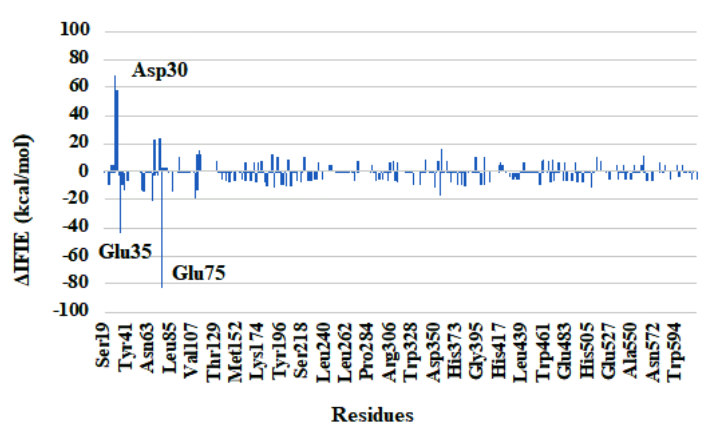

Fig. S2. Plots of differences in IFIE ( $\Delta$ IFIE) values for interfacial residues (summed up counter residues in RBD or ACE2) for three variants (UK, South Africa and Brazil). Numerical values of top 10 residues for simplicity are listed in Tables S2 and S3. 
PIEDA - residue by residue (Wuhan)

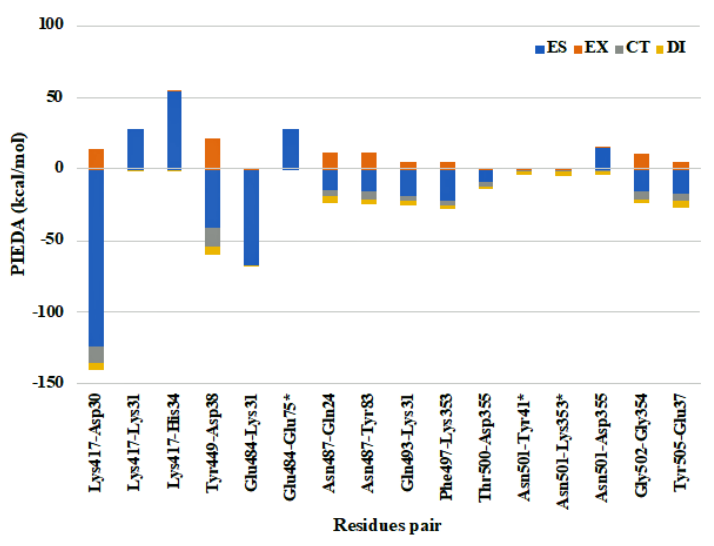

PIEDA - residue by residue (S.Africa)

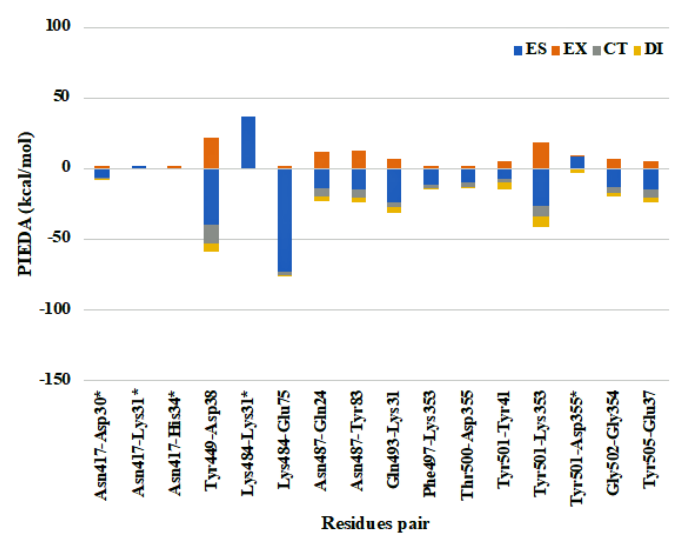

PIEDA - residue by residue (UK)

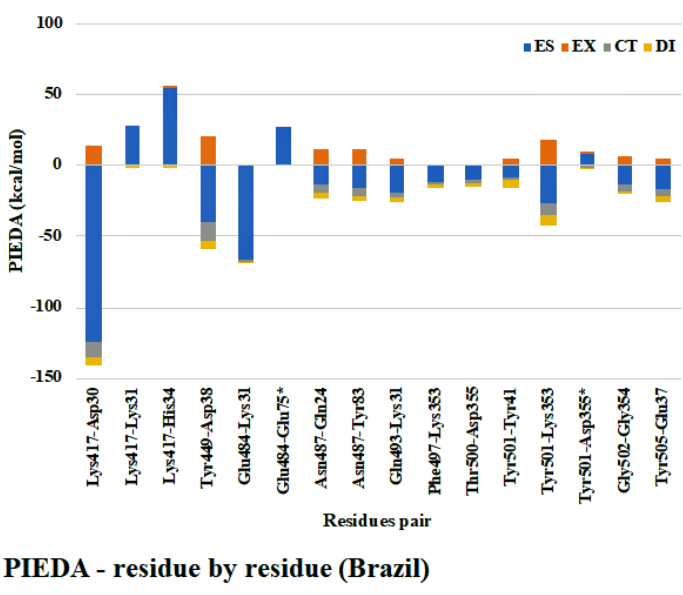

100 $-\mathrm{ES} \approx \mathrm{EX}=\mathrm{CT}=\mathrm{DI}$

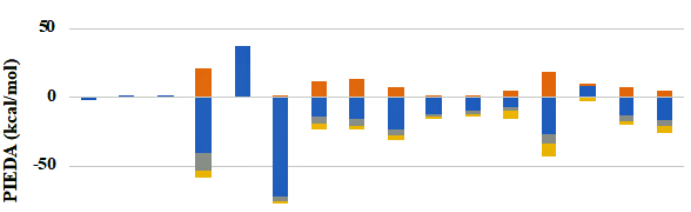

$-100$

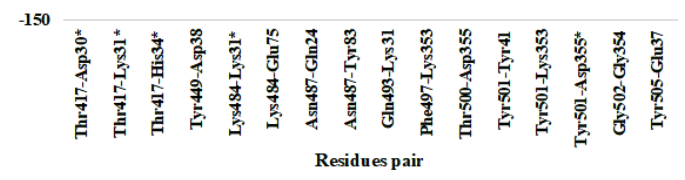

Fig. S3. Plots of PIEDA values (consisting of "ES", "EX", "CT" and "DI"). Pairs of residues correspond to those in Fig. 3. Asterisk specifies cases that absolute IFIE values are larger than $10 \mathrm{kcal} / \mathrm{mol}$ in other models. "ES" only plot is due to Dimer-ES approximation. ${ }^{20}$ Numerical values are compiled in Tables S4 - S7. 


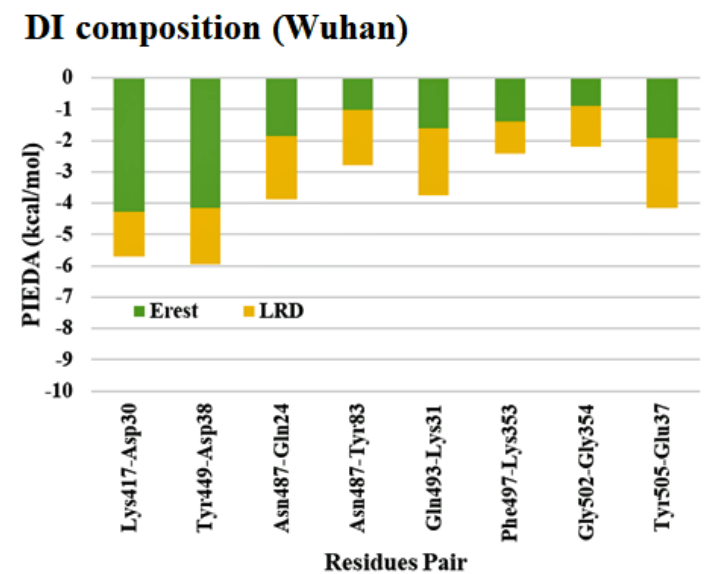

DI composition (S.Africa)

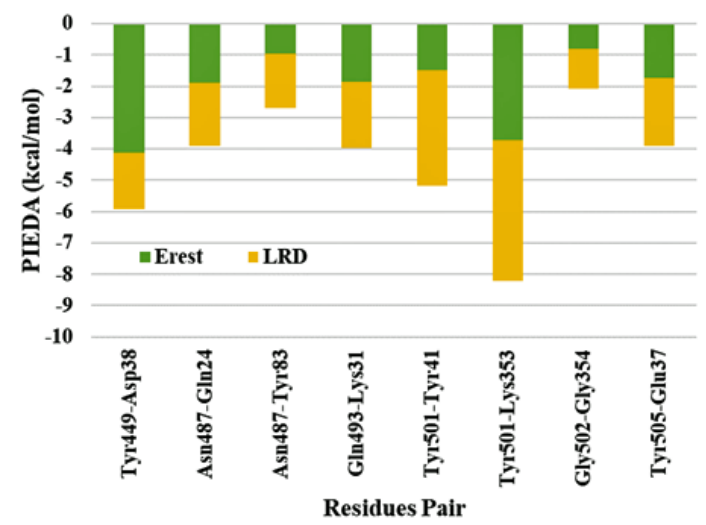

DI composition (UK)

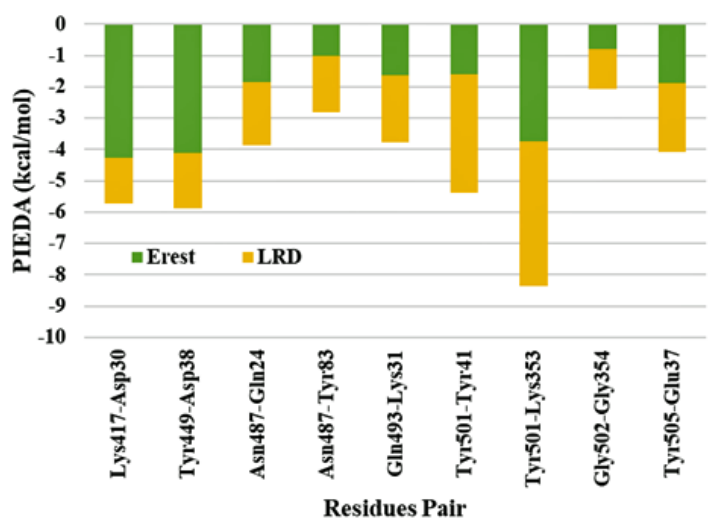

DI composition (Brazil)

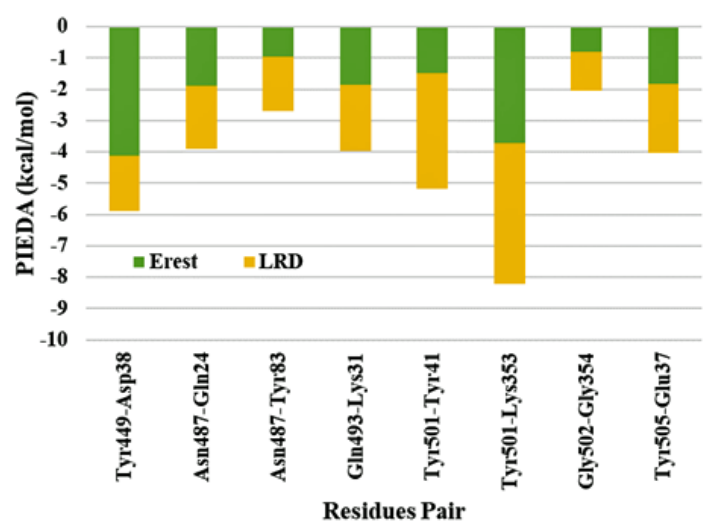

Fig. S4. Plots of decomposed "DI" contributions (more stable than $-2 \mathrm{kcal} / \mathrm{mol}$ ) appeared in Fig. S3. Refer to main texts for meanings of "LRD" and "Erest". Numerical values are compiled in Tables S8 - S11. 


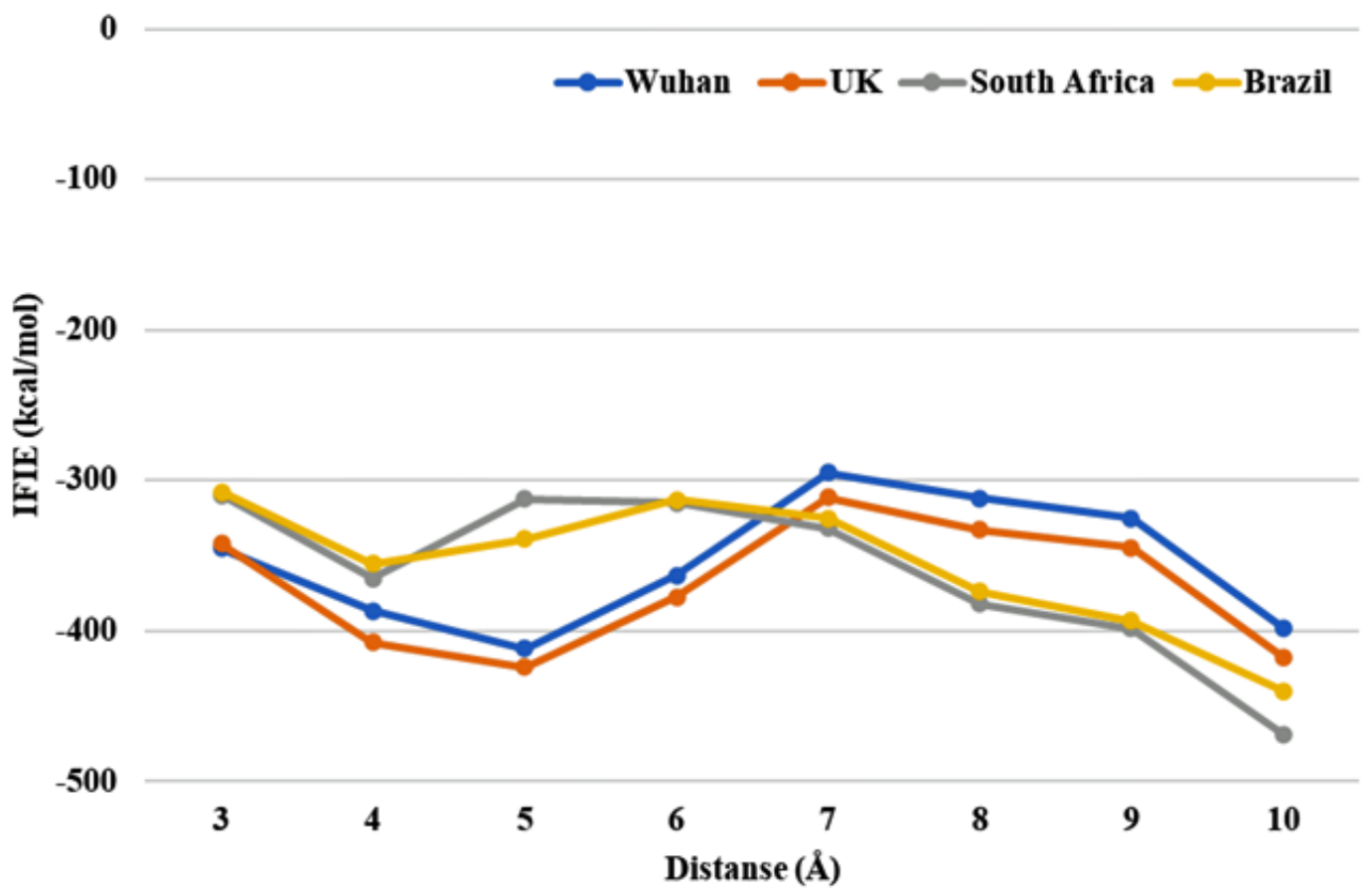

Fig. S5. Plots of partial summations IFIE from $3 \AA$ to $10 \AA$ (step $1 \AA$ ) at the interfacial region, the numbers of residue pairs between RBD and ACE2 are increased stepwise. Numerical values are compiled in Table S12. 
Table S1. Total IFIE values RBD - ACE2 interactions evaluated by various methods (see main texts). Unit in kcal/mol.

\begin{tabular}{ccccccc}
\hline & Basis & HF & MP2 & PR-MP2 & MP2.5 & MP3 \\
\hline \multirow{2}{*}{ Wuhan } & $6-31 \mathrm{G}^{*}$ & -766.0 & -891.6 & -886.7 & -879.6 & -867.5 \\
& cc-pVDZ & -773.2 & -914.3 & -908.3 & -900.5 & -886.7 \\
\multirow{2}{*}{ UK } & $6-31 \mathrm{G}^{*}$ & -792.3 & -925.2 & -920.3 & -912.3 & -899.3 \\
& cc-pVDZ & -794.7 & -943.4 & -937.1 & -928.6 & -913.8 \\
\multirow{3}{*}{ S Africa } & $6-31 \mathrm{G}^{*}$ & -977.0 & -1105.0 & -1100.1 & -1092.5 & -1080.1 \\
& cc-pVDZ & -979.9 & -1122.7 & -1116.6 & -1108.4 & -1094.1 \\
\multirow{2}{*}{ Brazil } & $6-31 \mathrm{G}^{*}$ & -976.2 & -1103.8 & -1098.9 & -1091.4 & -1079.0 \\
& cc-pVDZ & -978.7 & -1121.0 & -1115.0 & -1106.8 & -1092.6 \\
\hline
\end{tabular}


Table S2. Differences in IFIE ( $\Delta$ IFIE) for interfacial residues (summed up counter residues in ACE2) for three variants (UK, South Africa and Brazil). Values evaluated by MP2.5/cc-pVDZ method. Unit in kcal/mol.

\begin{tabular}{cccccc}
\hline \multicolumn{2}{c}{ UK } & \multicolumn{2}{c}{ South Africa } & \multicolumn{2}{c}{ Brazil } \\
\hline Residues & $\Delta$ IFIE & Residues & $\Delta$ IFIE & Residues & $\Delta$ IFIE \\
\hline Asn(Tyr)501 & -35.8 & Glu(Lys)484 & -446.5 & Glu(Lys)484 & -446.4 \\
Gln498 & 4.4 & Lys(Asn)417 & 276.9 & Lys(Thr)417 & 283.4 \\
Gln506 & -2.0 & Asn(Tyr)501 & -35.3 & Asn(Tyr)501 & -35.3 \\
Gly502 & 1.6 & Phe456 & -6.3 & Phe456 & -6.4 \\
Phe497 & 1.6 & Gln498 & 4.6 & Gln498 & 4.6 \\
Tyr505 & 1.3 & Arg403 & 3.6 & Asp420 & -3.1 \\
Val503 & 1.1 & Asp420 & -2.9 & Gln493 & -2.8 \\
Gly496 & 0.7 & Gln493 & -2.8 & Leu455 & -2.6 \\
Pro499 & 0.6 & Leu455 & -2.5 & Gly502 & 2.2 \\
Arg403 & -0.4 & Tyr505 & 2.2 & Gln506 & -1.8 \\
\hline
\end{tabular}


Table S3. Differences in IFIE ( $\triangle$ IFIE) for interfacial residues (summed up counter residues in RBD) for three variants (UK, South Africa and Brazil). Values evaluated by MP2.5/cc-pVDZ method. Unit in kcal/mol.

\begin{tabular}{cccccc}
\hline & \multicolumn{2}{c}{ UK } & \multicolumn{2}{c}{ South Africa } & \multicolumn{2}{c}{ Brazil } \\
\hline Residues & $\Delta$ IFIE & Residues & $\Delta$ IFIE & Residues & $\Delta$ IFIE \\
\hline Asp355 & -7.7 & Glu75 & -83.3 & Glu75 & -83.0 \\
Tyr41 & -7.5 & Asp30 & 63.2 & Asp30 & 68.2 \\
Lys353 & -7.3 & Lys31 & 60.0 & Lys31 & 58.7 \\
Arg357 & 6.1 & Glu35 & -43.8 & Glu35 & -43.7 \\
Gln42 & -6.1 & Lys74 & 24.3 & Lys74 & 24.4 \\
Asp38 & 5.5 & Lys68 & 23.3 & Lys68 & 23.4 \\
Asp350 & -2.2 & Asp67 & -20.7 & Asp67 & -20.9 \\
His34 & -2.0 & Glu110 & -19.8 & Glu110 & -19.8 \\
Glu329 & -1.6 & Asp355 & -16.3 & Asp355 & -17.3 \\
Glu57 & -1.4 & Lys114 & 15.6 & Arg357 & 16.3 \\
\hline
\end{tabular}


Table S4. PIEDA values of original Wuhan as reference. Values evaluated by MP2.5/ccpVDZ method. Energy unit in $\mathrm{kcal} / \mathrm{mol}$, and distance (Dist.) unit in Å. Asterisk specifies cases that absolute IFIE values are larger than $10 \mathrm{kcal} / \mathrm{mol}$ in other models.

\begin{tabular}{ccccccc}
\hline Residue Pair & & & & & & \\
(Spike RBD - ACE2) & Dist. & IFIE & ES & EX & CT & DI \\
\hline Lys417-Asp30 & 1.7 & -126.9 & -124.5 & 13.7 & -10.4 & -5.7 \\
Lys417-Lys31 & 5.1 & 28.7 & 28.8 & 0.0 & 0.0 & -0.1 \\
Lys417-His34 & 3.4 & 54.5 & 55.0 & 0.0 & -0.2 & -0.5 \\
Tyr449-Asp38 & 1.6 & -37.6 & -40.6 & 22.2 & -13.2 & -5.9 \\
Glu484-Lys31 & 3.7 & -67.9 & -66.9 & 0.0 & -0.6 & -0.5 \\
Glu484-Glu75* & 8.7 & 27.9 & 27.9 & 0.0 & 0.0 & 0.0 \\
Asn487-Gln24 & 1.9 & -10.8 & -13.9 & 12.1 & -5.2 & -3.9 \\
Asn487-Tyr83 & 1.7 & -12.0 & -15.5 & 12.4 & -6.1 & -2.8 \\
Gln493-Lys31 & 2.2 & -20.0 & -18.8 & 5.6 & -2.9 & -3.8 \\
Phe497-Lys353 & 2.1 & -22.5 & -22.6 & 5.3 & -2.7 & -2.4 \\
Thr500-Asp355 & 2.9 & -13.3 & -9.1 & 0.3 & -2.9 & -1.5 \\
Asn501-Tyr41* & 3.2 & -2.9 & 0.0 & 0.5 & -1.4 & -2.1 \\
Asn501-Lys353* & 2.9 & -3.4 & -0.1 & 0.6 & -1.6 & -2.4 \\
Asn501-Asp355 & 2.7 & 12.7 & 14.9 & 0.7 & -1.5 & -1.5 \\
Gly502-Gly354 & 1.8 & -12.4 & -15.6 & 11.1 & -5.7 & -2.2 \\
Tyr505-Glu37 & 2.0 & -21.6 & -17.1 & 5.0 & -5.3 & -4.2 \\
\hline
\end{tabular}


Table S5. PIEDA values of UK variant. Values evaluated by MP2.5/cc-pVDZ method. Energy unit in $\mathrm{kcal} / \mathrm{mol}$, and distance (Dist.) unit in $\AA$. Asterisk specifies cases that absolute IFIE values are larger than $10 \mathrm{kcal} / \mathrm{mol}$ in other models.

\begin{tabular}{ccccccc}
\hline Residue Pair & & & & & & \\
(Spike RBD - ACE2) & Dist. & IFIE & ES & EX & CT & DI \\
\hline Lys417-Asp30 & 1.7 & -126.9 & -124.5 & 13.7 & -10.4 & -5.7 \\
Lys417-Lys31 & 5.1 & 28.7 & 28.8 & 0.0 & 0.0 & -0.1 \\
Lys417-His34 & 3.4 & 54.5 & 55.1 & 0.0 & -0.2 & -0.5 \\
Tyr449-Asp38 & 1.6 & -37.4 & -40.0 & 21.3 & -12.9 & -5.9 \\
Glu484-Lys31 & 3.7 & -68.0 & -67.0 & 0.0 & -0.6 & -0.5 \\
Glu484-Glu75* & 8.7 & 28.0 & 28.0 & 0.0 & 0.0 & 0.0 \\
Asn487-Gln24 & 1.9 & -10.8 & -13.9 & 12.1 & -5.2 & -3.9 \\
Asn487-Tyr83 & 1.7 & -12.0 & -15.5 & 12.4 & -6.1 & -2.8 \\
Gln493-Lys31 & 2.2 & -20.1 & -18.9 & 5.6 & -3.0 & -3.8 \\
Phe497-Lys353 & 2.8 & -14.6 & -11.8 & 0.6 & -2.1 & -1.4 \\
Thr500-Asp355 & 3.0 & -13.9 & -9.7 & 0.2 & -3.0 & -1.5 \\
Tyr501-Tyr41 & 2.5 & -10.6 & -7.4 & 5.2 & -3.1 & -5.4 \\
Tyr501-Lys353 & 1.8 & -23.9 & -27.0 & 18.9 & -7.4 & -8.4 \\
Tyr501-Asp355* & 2.7 & 6.0 & 8.2 & 0.6 & -1.3 & -1.5 \\
Gly502-Gly354 & 2.0 & -13.1 & -13.1 & 7.1 & -4.9 & -2.1 \\
Tyr505-Glu37 & 2.0 & -20.9 & -16.5 & 4.9 & -5.2 & -4.1 \\
\hline
\end{tabular}


Table S6. PIEDA values of South Africa variant. Values evaluated by MP2.5/cc-pVDZ method. Energy unit in kcal/mol, and distance (Dist.) unit in $\AA$. Asterisk specifies cases that absolute IFIE values are larger than $10 \mathrm{kcal} / \mathrm{mol}$ in other models.

\begin{tabular}{ccccccc}
\hline $\begin{array}{c}\text { Residue Pair } \\
\text { (Spike RBD - }\end{array}$ & Dist. & IFIE & ES & EX & CT & DI \\
ACE2) & & & & & & \\
\hline Asn417-Asp30* & 3.8 & -7.3 & -6.6 & 0.0 & -0.4 & -0.3 \\
Asn417-Lys31* & 6.7 & 1.3 & 1.3 & 0.0 & 0.0 & 0.0 \\
Asn417-His34* & 4.3 & 0.5 & 0.7 & 0.0 & -0.1 & -0.2 \\
Tyr449-Asp38 & 1.6 & -37.4 & -40.0 & 21.2 & -12.8 & -5.9 \\
Lys484-Lys31* & 5.0 & 36.4 & 36.4 & 0.0 & 0.0 & 0.0 \\
Lys484-Glu75 & 3.0 & -76.8 & -72.5 & 0.1 & -2.9 & -1.5 \\
Asn487-Gln24 & 1.9 & -11.5 & -14.3 & 11.9 & -5.2 & -3.9 \\
Asn487-Tyr83 & 1.7 & -11.5 & -15.1 & 12.3 & -6.0 & -2.7 \\
Gln493-Lys31 & 2.1 & -24.9 & -23.8 & 6.5 & -3.6 & -4.0 \\
Phe497-Lys353 & 2.8 & -14.6 & -11.8 & 0.6 & -2.1 & -1.4 \\
Thr500-Asp355 & 3.0 & -13.9 & -9.7 & 0.2 & -3.0 & -1.5 \\
Tyr501-Tyr41 & 2.5 & -10.6 & -7.2 & 4.8 & -3.0 & -5.2 \\
Tyr501-Lys353 & 1.8 & -23.8 & -26.8 & 18.4 & -7.2 & -8.2 \\
Tyr501-Asp355* & 2.8 & 6.1 & 8.3 & 0.6 & -1.2 & -1.5 \\
Gly502-Gly354 & 2.0 & -13.0 & -12.9 & 6.9 & -4.9 & -2.1 \\
Tyr505-Glu37 & 2.0 & -19.9 & -15.6 & 4.4 & -4.9 & -3.9 \\
\hline
\end{tabular}


Table S7. PIEDA values of Brazil variant. Values evaluated by MP2.5/cc-pVDZ method. Energy unit in $\mathrm{kcal} / \mathrm{mol}$, and distance (Dist.) unit in $\AA$. Asterisk specifies cases that absolute IFIE values are larger than $10 \mathrm{kcal} / \mathrm{mol}$ in other models.

\begin{tabular}{ccccccc}
\hline Residue Pair & & & & & & \\
(Spike RBD - ACE2) & Dist. & IFIE & ES & EX & CT & DI \\
\hline Thr417-Asp30* & 5.5 & -1.6 & -1.6 & 0.0 & 0.0 & 0.0 \\
Thr417-Lys31* & 7.6 & 0.3 & 0.3 & 0.0 & 0.0 & 0.0 \\
Thr417-His34* & 3.7 & -0.3 & 0.0 & 0.0 & -0.1 & -0.1 \\
Tyr449-Asp38 & 1.6 & -37.3 & -39.9 & 21.2 & -12.7 & -5.9 \\
Lys484-Lys31* & 5.0 & 36.3 & 36.3 & 0.0 & 0.0 & 0.0 \\
Lys484-Glu75 & 3.0 & -76.8 & -72.5 & 0.1 & -2.9 & -1.5 \\
Asn487-Gln24 & 1.9 & -11.5 & -14.3 & 11.9 & -5.2 & -3.9 \\
Asn487-Tyr83 & 1.7 & -11.5 & -15.1 & 12.3 & -6.0 & -2.7 \\
Gln493-Lys31 & 2.1 & -25.0 & -24.0 & 6.5 & -3.6 & -4.0 \\
Phe497-Lys353 & 2.8 & -14.7 & -11.8 & 0.6 & -2.1 & -1.4 \\
Thr500-Asp355 & 3.0 & -13.9 & -9.7 & 0.2 & -3.0 & -1.5 \\
Tyr501-Tyr41 & 2.5 & -10.6 & -7.2 & 4.8 & -3.0 & -5.2 \\
Tyr501-Lys353 & 1.8 & -23.9 & -26.9 & 18.4 & -7.3 & -8.2 \\
Tyr501-Asp355* & 2.8 & 6.1 & 8.2 & 0.6 & -1.2 & -1.5 \\
Gly502-Gly354 & 2.0 & -12.9 & -12.8 & 6.8 & -4.9 & -2.1 \\
Tyr505-Glu37 & 2.0 & -20.4 & -16.1 & 4.8 & -5.0 & -4.0 \\
\hline
\end{tabular}


Table S8. Decomposed "DI" contributions (more stable than $-2 \mathrm{kcal} / \mathrm{mol}$ ) of original Wuhan. Values evaluated by MP2.5/cc-pVDZ method. Unit in kcal/mol.

\begin{tabular}{cccc}
\hline $\begin{array}{c}\text { Residue Pair } \\
\text { (Spike RBD - ACE2) }\end{array}$ & DI & LRD & Erest \\
\hline Lys417-Asp30 & -5.7 & -1.4 & -4.3 \\
Tyr449-Asp38 & -5.9 & -1.8 & -4.1 \\
Asn487-Gln24 & -3.9 & -2.0 & -1.9 \\
Asn487-Tyr83 & -2.8 & -1.8 & -1.0 \\
Gln493-Lys31 & -3.8 & -2.1 & -1.6 \\
Phe497-Lys353 & -2.4 & -1.1 & -1.4 \\
Gly502-Gly354 & -2.2 & -1.3 & -0.9 \\
Tyr505-Glu37 & -4.2 & -2.2 & -1.9 \\
\hline
\end{tabular}


Table S9. Decomposed "DI" contributions (more stable than $-2 \mathrm{kcal} / \mathrm{mol}$ ) of UK variant.

Values evaluated by MP2.5/cc-pVDZ method. Unit in $\mathrm{kcal} / \mathrm{mol}$.

\begin{tabular}{cccc}
\hline $\begin{array}{c}\text { Residue Pair } \\
\text { (Spike RBD - ACE2) }\end{array}$ & DI & LRD & Erest \\
\hline Lys417-Asp30 & -5.7 & -1.4 & -4.3 \\
Tyr449-Asp38 & -5.9 & -1.8 & -4.1 \\
Asn487-Gln24 & -3.9 & -2.0 & -1.9 \\
Asn487-Tyr83 & -2.8 & -1.8 & -1.0 \\
Gln493-Lys31 & -3.8 & -2.1 & -1.6 \\
Tyr501-Tyr41 & -5.4 & -3.8 & -1.6 \\
Tyr501-Lys353 & -8.4 & -4.6 & -3.7 \\
Gly502-Gly354 & -2.1 & -1.3 & -0.8 \\
Tyr505-Glu37 & -4.1 & -2.2 & -1.9 \\
\hline
\end{tabular}


Table S10. Decomposed "DI" contributions (more stable than $-2 \mathrm{kcal} / \mathrm{mol}$ ) of South Africa variant. Values evaluated by MP2.5/cc-pVDZ method. Unit in kcal/mol.

\begin{tabular}{cccc}
\hline $\begin{array}{c}\text { Residue Pair } \\
\text { (Spike RBD - ACE2) }\end{array}$ & DI & LRD & Erest \\
\hline Tyr449-Asp38 & -5.9 & -1.8 & -4.1 \\
Asn487-Gln24 & -3.9 & -2.0 & -1.9 \\
Asn487-Tyr83 & -2.7 & -1.8 & -1.0 \\
Gln493-Lys31 & -4.0 & -2.1 & -1.9 \\
Tyr501-Tyr41 & -5.2 & -3.7 & -1.5 \\
Tyr501-Lys353 & -8.2 & -4.5 & -3.7 \\
Gly502-Gly354 & -2.1 & -1.3 & -0.8 \\
Tyr505-Glu37 & -3.9 & -2.2 & -1.7 \\
\hline
\end{tabular}


Table S11. Decomposed "DI" contributions (more stable than $-2 \mathrm{kcal} / \mathrm{mol}$ ) of Brazil variant. Values evaluated by MP2.5/cc-pVDZ method. Unit in kcal/mol.

\begin{tabular}{cccc}
\hline $\begin{array}{c}\text { Residue Pair } \\
\text { (Spike RBD - ACE2) }\end{array}$ & DI & LRD & Erest \\
\hline Tyr449-Asp38 & -5.9 & -1.8 & -4.1 \\
Asn487-Gln24 & -3.9 & -2.0 & -1.9 \\
Asn487-Tyr83 & -2.7 & -1.8 & -1.0 \\
Gln493-Lys31 & -4.0 & -2.1 & -1.9 \\
Tyr501-Tyr41 & -5.2 & -3.7 & -1.5 \\
Tyr501-Lys353 & -8.2 & -4.5 & -3.7 \\
Gly502-Gly354 & -2.1 & -1.3 & -0.8 \\
Tyr505-Glu37 & -4.0 & -2.2 & -1.8 \\
\hline
\end{tabular}


Table S12. Partial summations IFIE from $3 \AA$ to $10 \AA$ (step $1 \AA$ ) at the interfacial region.

Values evaluated by MP2.5/cc-pVDZ method. Unit in $\mathrm{kcal} / \mathrm{mol}$.

\begin{tabular}{ccccccccc}
\hline Dist.(Å) & 3 & 4 & 5 & 6 & 7 & 8 & 9 & 10 \\
\hline Wuhan & -345.4 & -387.0 & -412.0 & -363.3 & -294.9 & -312.0 & -325.1 & -398.3 \\
UK & -341.9 & -407.9 & -424.0 & -377.6 & -311.6 & -333.1 & -344.9 & -417.5 \\
S.Africa & -309.9 & -365.4 & -312.3 & -314.8 & -331.8 & -382.3 & -398.4 & -469.0 \\
Brazil & -307.9 & -355.4 & -339.0 & -313.1 & -325.1 & -374.1 & -393.2 & -439.9 \\
\hline
\end{tabular}

